\title{
Exposure to Heavy Metal Elements May Significantly Increase Serum Prostate-Specific Antigen Levels With Overdosed Dietary Zinc
}

\section{Xiaoyue Chen}

Guizhou University

\section{Chi Chen}

Guiyang college of triditional chinese medicine

Jukun Song

Guizhou Provincial People's Hospital

Heng Liu

Zunyi Medical University

\section{Lingyue An}

Guizhou Provincial People's Hospital

\section{Dongbo Yuan}

Guizhou Provincial People's Hospital

\section{Guohua Zhu}

Guizhou Medical University

\section{Bin $\mathrm{Hu}$}

Zunyi Medical University

\section{Wei Wang}

Guizhou Provincial People's Hospital

Zhangcheng Liu

Guizhou Provincial People's Hospital

Ke Jiao

Guizhou Provincial People's Hospital

Hao Su

Guizhou Provincial People's Hospital

Chang Zhang

Guizhou Provincial People's Hospital

\section{Fan Zhang}

Guizhou Provincial People's Hospital

\section{Kehua Jiang}

Guizhou Provincial People's Hospital

Fu Luo 
Guizhou Provincial People's Hospital

\section{Zongjian Tan}

Guizhou Provincial People's Hospital

\section{Bohao Zheng}

Guizhou Provincial People's Hospital

\section{Zhaolin Sun}

Guizhou Provincial People's Hospital

\section{Weiming Chen}

Guizhou Provincial People's Hospital

\section{Fa Sun}

Guizhou Provincial People's Hospital

jianguo zhu ( $\nabla$ doctorzhujianguo@163.com )

Guizhou Provincial People's Hospital https://orcid.org/0000-0002-1171-0126

\section{Research}

Keywords: Lead, cadmium, mercury, zinc intake, prostate-specific antigen (PSA), NHANES

Posted Date: April 27th, 2021

DOI: https://doi.org/10.21203/rs.3.rs-437706/v1

License: (c) (i) This work is licensed under a Creative Commons Attribution 4.0 International License. Read Full License 


\section{Abstract}

Background: Serum prostate-specific antigen (PSA) is a primary metric for diagnosis and prognosis of prostate cancer ( $\mathrm{PCa}$ ). Exposure to heavy metals, such as lead, cadmium, mercury, and zinc can impact PSA levels in PCa patients. However, it is unclear whether this effect also occurs in men without PCa, which may lead to the overdiagnosis of PCa.

Method: Data on a total of 5,089 American men who had never been diagnosed with PCa were obtained from the National Health and Nutrition Examination Survey performed from 2003-2010. The relationship between serum PSA levels (dependent variable) and concentrations of lead ( $\mu \mathrm{mol} / \mathrm{L})$, cadmium (nmol/L), and mercury $(\mu \mathrm{mol} / \mathrm{L})$ were investigated with dietary zinc intake being used as a potential modifier or covariate in a weighted linear regression model and a generalized additive model. A series of bootstrapping analyses were performed to evaluate sensitivity and specificity using these models.

Results: Regression analyses suggested that, in general, lead, cadmium, or mercury did not show an association with PSA levels, which was consistent with the results of the bootstrapping analyses. However, in a subgroup of participants with a high level of dietary zinc intake ( $\geq 14.12 \mathrm{mg} /$ day), a significant positive association between cadmium and serum PSA was identified $(1.06,95 \% \mathrm{Cl}, \mathrm{P}=0.0268$, $P$ for interaction $=0.0249$ ).

Conclusions: With high-level zinc intake, serum PSA levels may rise in PCa-free men as the exposure to cadmium increases, leading to a potential risk of an overdiagnosis of PCa and unnecessary treatment. Therefore, environmental variables should be factored in the current diagnostic model for PCa that is solely based on PSA measurements. Different criteria for PSA screening are necessary based on geographical variables. Further investigations are needed to uncover the biological and biochemical relationship between zinc, cadmium, and serum PSA levels to more precisely diagnose PCa.

\section{Introduction}

The latest data from the United States Cancer Statistics (USCS) program at the US Centers for Disease Control and Prevention (UCDCP) indicate that prostate cancer (PCa) is the most common cancer among men in the US [1]. There were a total of 3,650,030 PCa cases in 2019, which is more than threefold that of colorectal cancer (994,210 cases), the second most common cancer in men [3]. In 2030, the estimated number of PCa patients will be greater than that for female breast cancer patients. In each subsequent year, there will be about 164,960 new PCa cases, among which 29,430 patients will die from the disease, which has a high mortality rate of $17.84 \%$ [2]. A global study found that the incidence and mortality of $\mathrm{PCa}$ are higher in the US than in other regions and countries [4-5]. Interestingly, data show that the incidence of PCa in US male adults or in male Japanese immigrants are significantly higher than in males in Japan or Korea [6-7]. This suggests that the environment might play a significant role in causing PCa. 
Prostate-specific antigen (PSA), a member of the kallikrein-related peptidase family, is secreted in small amounts in the serum of normal males by prostate epithelial cells [8]. PCa screening based on the measurement of PSA levels using an annual PSA test was approved in 1995 by the US Food and Drug Administration (FDA), and these standards have been widely used in the clinic for PCa screening and surveillance [17]. Studies have shown that the serum PSA level is not a strong indicator for the presence of PCa, with only a $24 \%$ positive predictive value [9-10]; this is because serum PSA levels are subject to many factors, including disease, age, smoking status, alcohol intake, as well as others [11-16], which may interfere with a definitive diagnosis of PCa. Nevertheless, Tikkinen et al. still recommend using serum PSA as a criterion for PCa screening and surveillance, which has positive implications for early detection and prevention of the disease [17].

Many secondary analyses based on the National Health and Nutrition Examination Survey (NHANES) showed that drugs, food, and metals can impact PSA levels in serum. However, no studies have investigated associations between heavy metals and PSA levels in a large sample size. Non-essential heavy metal elements, such as lead, cadmium, and mercury, are distributed in rocks and other environments and can be transferred to the human body through depositional amplification in the food chain. As reported, underground workers with increased lead exposure through the food chain and air transfer had a significantly higher incidence of immunotoxicity [18]. The lead exposure in the living environment is mainly because of the pollution from plastics, pigments, and other sources [19-20]. In the manufacturing industry, $78 \%$ of the cadmium exposure is because of the pollution from batteries, which also poses a threat to the environment. The target organs for cadmium deposition include the liver and kidneys, where cadmium binds to metallothionein proteins and is eliminated, limiting its damage to the human body [21]. It has been shown that cadmium exposure is significantly associated with various types of cancer [22]. A study based on a group of 295 men over the age of 50 years reported a negative correlation between concentrations of serum cadmium and PSA levels [23]. The only research on mercury sediment in the food chain has shown that the deposition of mercury in the blood of semipalmated sandpipers, small shorebirds, did not change with age [24]. The mechanism by which mercury may promote cancer could be related to interstitial communication between gap junctions and the involvement of inflammatory cytokines [25].

Zinc, an essential heavy metal, has been reported to play an important role in human health. For example, the earliest literature from 1786 reported that zinc had beneficial effects in epilepsy [26]. Ogawa et al. found that skin was highly enriched in zinc (ranked third among human organs), and zinc deficiency in skin was related to various disorders, such as acrodermatitis enteropathica [27]. Research has shown that zinc can help accelerate the wound-healing processes [28]. Moreover, zinc has been shown to be an effective treatment for male infertility [29], impotence [30], and chronic prostatitis [31]. Recent studies found that there was an association between low-level zinc metabolism and tumorigenesis [32-34]. A study based on 1,961 Spanish men showed that excessive zinc intake led to increased PSA levels, but no association between serum zinc and prostate cancer was detected [35]. Nevertheless, blood zinc levels in patients with early prostatitis were significantly higher than in normal subjects [36-37]. A study based on only 57 men between the ages of 21-40 years showed no significant association between levels of lead, 
cadmium, or zinc, and PSA levels in blood [38], while a study on 1,320 male participants over 40 years old using data from NHANES came to the same conclusion [39]. To date, data that evaluate the relationship between mercury and PSA levels have not been generated.

To reduce the potential bias from studies based on small sample sizes, we performed the current study using data on 5,089 participants from NHANES to investigate the association between lead, cadmium, mercury, or zinc intake levels (recorded on the first day on the survey record) with PSA levels in American males over the age of 40 . The results indicated that given a high level of dietary zinc, serum PSA levels may rise in PCa-free males as exposure to cadmium increases.

\section{Materials And Methods}

\section{Data source}

NHANES is a program designed to assess the health and nutritional status of adults and children in the US. NHANES includes data on demographic, socioeconomic, dietary, and health-related issues. NHANES includes medical, dental, and physiological measurements including height, weight, and blood pressure, all based on national standards, with all laboratory tests conducted by trained medical personnel. Data and information acquired by the program are being used in epidemiological and health science research to (1) assess nutritional status and its relationship with health promotion and disease prevention, and (2) determine the prevalence and risk factors of major diseases. The results of the research will help to develop public health policies and also help guide designing health plans and services. More detailed information about this data resource may be found at http://www.cdc.gov/nchs/nhanes/nhanes_questionnairees.htm.

\section{Study population}

The NHANES database contains PSA data collected from 2003-2010. We integrated this data from the four biennial NHANES survey cycles (2003-2004, 2005-2006, 2007-2008, and 2009-2010) and conducted a secondary data analysis. We only considered males age 40 and over in the NHANES database. Men with PCa, prostatitis or recent prostate surgery (i.e., rectal examination within a week, and prostate biopsy, surgery, or cystoscopy within a month) were not included in the study. We also excluded men who used 5-alpha- reductase inhibitors or other hormone therapies (such as testosterone replacement or medical castration), or whose clinical or social demographic data were incomplete. A total of 5,089 men out of 41,156 participants in NHANES were finally included in our study (See flow chart in Fig. 1).

\section{Variables}

In this study, the independent variables were lead exposure ( $\mu \mathrm{mol} / \mathrm{L})$, cadmium exposure (nmol/L), mercury exposure ( $\mu \mathrm{mol} / \mathrm{L})$, and dietary zinc intake (mg/day); the target dependent variable was PSA $(\mathrm{ng} / \mathrm{mL})$. Covariates that may have impacted PSA levels (based on the literature) were selected from the 
NHANES database, including continuous and categorical variables. Continuous variables included age, poverty income ratio, alanine aminotransferase $(A L T ; \mu / L)$, aspartate aminotransferase $(A S T ; \mu / L)$, serum urea nitrogen (BUN, $\mathrm{mg} / \mathrm{dL})$, serum creatinine $(\mathrm{Cr}, \mathrm{mg} / \mathrm{dL})$, serum uric acid $(\mathrm{UA}, \mathrm{mg} / \mathrm{dL})$, body mass index $\left(\mathrm{kg} / \mathrm{m}_{2}\right)$, dietary foods [alcohol $(\mathrm{mg})$, caffeine $(\mathrm{mg})$, calcium $(\mathrm{mg})$, and GM $(\mathrm{gm})$ ], saturated fiber $(\mathrm{mg})$, energy $(\mathrm{kJ})$, body fatty acid $(\mathrm{mg})$, total polyunsaturated fatty acid, total fatty acid (gm), and total carbohydrate fatty acid (measured using C-reactive protein).

Categorical variables included hypertension history (no/yes), diabetes history (no/yes), DMDEDUC2 (lower than high school, high school, higher than high school), DMDMARTL (married, living with partner versus not, single), coronary heart disease (no/yes), stroke (yes/no), race/ethnicity (Mexican American, other Hispanic American, non-Hispanic white, non-Hispanic black, other race), smoked at least 100 cigarettes during lifetime (no/yes), enlarged prostate (no/yes), and average level of physical activity each day (PAQ180) (daily exercise: none, slight, normal, vigorous). All variables, which were derived from the participants' self-reports and used in the analysis, are summarized in Table 1.

\section{Data analysis}

The skewed distribution of blood lead, cadmium, mercury exposure, zinc intake, and PSA levels were log2 transformed prior to the analysis. Four subgroups were formed according to PSA levels: Q1 (0.07-0.577), Q2 (0.58-0.96), Q3 (0.97-1.88), and Q4 (1.89-74.02). Continuous variables were expressed as $\beta$ (95\% $\mathrm{Cl})$, and categorized variables were expressed as percentages. Data analysis was based on the following steps: (1) Univariate analysis, (2) multivariate analysis, (3) fully adjusted model, and (4) generalized additive model (GAM). The variables modified in the fully adjusted model included hypertension history, body mass index $\left(\mathrm{kg} / \mathrm{m}^{2}\right)$, diabetes history, DMDEDUC2.NEW NEW, DMDMARTL.NEW NEW, alcohol (gm) first day, high-density lipoprotein (HDL), poverty income ratio, enlarged prostate, LBDLDL, C-reactive protein $(\mathrm{mg} / \mathrm{dL})$, glycohemoglobin $(\%)$, triglycerides $(\mathrm{mg} / \mathrm{dL})$, coronary heart disease, stroke, PAQ180, age, race/ethnicity, smoked at least 100 cigarettes during lifetime, vitamin D (VITD), and zinc (mg) first day. The GAM was used to identify the non-linear relationship for the non-equidistant variation in PSA levels after adjustments for hypertension history, body mass index $\left(\mathrm{kg} / \mathrm{m}^{2}\right)$ (smooth), diabetes history, DMDEDUC, DMDMARTL, alcohol (gm) first day (smooth), HDL (smooth), poverty income ratio, enlarged prostate, LBDLDL (smooth), C-reactive protein (mg/dL) (smooth), glycohemoglobin (\%) (smooth), triglycerides (mg/dL) (smooth), coronary heart disease, stroke, PAQ180, age (year) (smooth), race/ethnicity, smoked at least 100 cigarettes during lifetime, VITD (smooth), and zinc (mg) first day (smooth). For each of the four metals, if the non-linear correlation model was observed, a two-piecewise linear regression model was performed to calculate the inflection point (or threshold effect) of the curve using a recursive method based on a maximum likelihood model [40]. Finally, a subgroup analysis was performed using stratified linear regression models with different zinc intake dose levels (low, medium, and high). The modifications and interactions of each subgroup, including lead, cadmium, and mercury with PSA levels, were tested using the likelihood ratio test. Data analysis was performed based on the $\mathrm{R}$ package. A $P$ value less than 0.05 was considered statistically significant. 


\section{Results}

\section{Basic participant characteristics}

Table 1 shows basic participant characteristics, including daily zinc intake $(\mathrm{mg})$ on the first day of the survey, and lead, cadmium, and mercury exposure for the 5,089 participants recruited according to the inclusion criteria. There were no differences between the four subgroups regarding the following variables: cadmium, LBDLDL, mercury, poverty income ratio, VITD, coronary heart disease, and smoking habit (smoked at least 100 cigarettes during lifetime). Significant differences were observed between the four subgroups in the following variables: body mass index $\left(\mathrm{kg} / \mathrm{m}^{2}\right)$, lead exposure $(\mu \mathrm{mol} / \mathrm{L})$, alcohol (mg) in first day, HDL, C-reactive protein (mg/dL), glycohemoglobin (\%), triglycerides ( $\mathrm{mg} / \mathrm{dL})$, age (years), zinc intake first day (mg), hypertension history, diabetes history, DMDEDU, DEDMARTL, stroke, race/ethnicity, enlarged prostate, and PAQ180. 
Table 1

Baseline characteristics of participants.

\begin{tabular}{|c|c|c|c|c|c|}
\hline $\begin{array}{l}P S A(\mathrm{ng} / \mathrm{ml}) \log 2 \\
\text { transform }\end{array}$ & Q1 & Q2 & Q3 & Q4 & $P$-value \\
\hline $\mathrm{N}$ & 1272 & 1272 & 1273 & 1272 & \\
\hline $\begin{array}{l}\text { Body mass } \\
\text { index, } \mathrm{Kg} / \mathrm{m} 2\end{array}$ & $\begin{array}{l}29.71(6.44) \\
28.72(15.91- \\
130.21)\end{array}$ & $\begin{array}{l}28.93(5.52) \\
28.15(14.59- \\
66.16)\end{array}$ & $\begin{array}{l}28.23(4.81) \\
27.83(15.66- \\
51.91)\end{array}$ & $\begin{array}{l}28.16(5.17) \\
27.74(14.20- \\
58.33)\end{array}$ & $\begin{array}{l}< \\
0.001^{\# \#}\end{array}$ \\
\hline Lead (umol/L) & $\begin{array}{l}0.12(0.09) \\
0.10(0.01- \\
1.23)\end{array}$ & $\begin{array}{l}0.12(0.11) \\
0.10(0.02- \\
1.46)\end{array}$ & $\begin{array}{l}0.12(0.09) \\
0.10(0.01- \\
1.08)\end{array}$ & $\begin{array}{l}0.14(0.11) \\
0.11(0.02- \\
1.59)\end{array}$ & $\begin{array}{l}< \\
0.001^{\# \#}\end{array}$ \\
\hline $\begin{array}{l}\text { Cadmium } \\
\text { (nmol/L) }\end{array}$ & $\begin{array}{l}5.02(5.25) \\
3.11(0.89- \\
41.82)\end{array}$ & $\begin{array}{l}5.10(6.09) \\
2.85(0.89- \\
78.29)\end{array}$ & $\begin{array}{l}5.12(5.63) \\
3.47(0.89- \\
76.07)\end{array}$ & $\begin{array}{l}5.19(4.73) \\
3.56(0.89- \\
43.60)\end{array}$ & 0.862 \\
\hline $\begin{array}{l}\text { Mercury, total } \\
\text { (umol/L) }\end{array}$ & $\begin{array}{l}8.61(12.54) \\
4.50(0.50- \\
156.70)\end{array}$ & $\begin{array}{l}9.01(12.48) \\
5.14(0.50- \\
192.10)\end{array}$ & $\begin{array}{l}9.83(17.71) \\
5.00(0.50- \\
427.60)\end{array}$ & $\begin{array}{l}8.51(12.11) \\
4.99(0.50- \\
189.60)\end{array}$ & 0.027 \\
\hline $\begin{array}{l}\text { Alcohol (gm) first } \\
\text { day }\end{array}$ & $\begin{array}{l}15.26(38.20) \\
0.00(0.00- \\
400.00)\end{array}$ & $\begin{array}{l}15.06(33.21) \\
0.00(0.00- \\
246.20)\end{array}$ & $\begin{array}{l}16.18(38.23) \\
0.00(0.00- \\
337.00)\end{array}$ & $\begin{array}{l}11.10(26.11) \\
0.00(0.00- \\
169.70)\end{array}$ & $\begin{array}{l}< \\
0.001^{\# \#}\end{array}$ \\
\hline $\mathrm{HDL}$ & $\begin{array}{l}47.07 \text { (13.37) } \\
45.00(19.00- \\
133.00)\end{array}$ & $\begin{array}{l}47.66(13.81) \\
45.00(15.00- \\
144.00)\end{array}$ & $\begin{array}{l}49.37(14.87) \\
46.00(19.00- \\
141.00)\end{array}$ & $\begin{array}{l}50.21(15.13) \\
47.00(19.00- \\
179.00)\end{array}$ & $\begin{array}{l}< \\
0.001 \# \#\end{array}$ \\
\hline $\begin{array}{l}\text { Poverty income } \\
\text { ratio }\end{array}$ & $\begin{array}{l}2.69(1.63) \\
2.30(0.00- \\
5.00)\end{array}$ & $\begin{array}{l}2.81(1.64) \\
2.65(0.00- \\
5.00)\end{array}$ & $\begin{array}{l}2.81(1.61) \\
2.56(0.00- \\
5.00)\end{array}$ & $\begin{array}{l}2.66(1.57) \\
2.34(0.00- \\
5.00)\end{array}$ & $0.011^{\#}$ \\
\hline LBDLDL & $\begin{array}{l}116.98 \\
(34.57) \\
117.00 \\
(22.00- \\
247.00)\end{array}$ & $\begin{array}{l}119.95 \\
(33.78) \\
118.00(43.00- \\
266.00)\end{array}$ & $\begin{array}{l}120.26 \\
(36.19) \\
120.00(32.00- \\
261.00)\end{array}$ & $\begin{array}{l}117.06 \\
(36.12) \\
114.00(37.00- \\
289.00)\end{array}$ & 0.117 \\
\hline $\begin{array}{l}\text { C-reactive } \\
\text { protein }(\mathrm{mg} / \mathrm{dL})\end{array}$ & $\begin{array}{l}0.44(0.98) \\
0.19(0.01- \\
18.50)\end{array}$ & $\begin{array}{l}0.38(0.81) \\
0.19(0.01- \\
10.89)\end{array}$ & $\begin{array}{l}0.38(0.80) \\
0.18(0.01- \\
17.50)\end{array}$ & $\begin{array}{l}0.50(1.24) \\
0.22(0.01- \\
18.10)\end{array}$ & $\begin{array}{l}< \\
0.001^{\# \#}\end{array}$ \\
\hline $\begin{array}{l}\text { Glycohemoglobin } \\
(\%)\end{array}$ & $\begin{array}{l}5.96(1.33) \\
5.60(4.00- \\
15.50)\end{array}$ & $\begin{array}{l}5.82(1.15) \\
5.50(3.70- \\
14.00)\end{array}$ & $\begin{array}{l}5.89(1.18) \\
5.60(4.00- \\
18.00)\end{array}$ & $\begin{array}{l}5.89(1.01) \\
5.60(4.10- \\
13.50)\end{array}$ & $0.005^{\# \#}$ \\
\hline $\begin{array}{l}\text { Triglycerides } \\
(\mathrm{mg} / \mathrm{dL})\end{array}$ & $\begin{array}{l}184.22 \\
(187.52) \\
140.00 \\
(30.00- \\
2566.00)\end{array}$ & $\begin{array}{l}180.48 \\
(152.00) \\
138.00(24.00- \\
2693.00)\end{array}$ & $\begin{array}{l}166.48 \\
(125.89) \\
129.00(33.00- \\
1223.00)\end{array}$ & $\begin{array}{l}153.48 \\
(107.25) \\
125.00(21.00- \\
1460.00)\end{array}$ & $\begin{array}{l}< \\
0.001^{\# \#}\end{array}$ \\
\hline \multicolumn{6}{|c|}{$\begin{array}{l}\text { HDL, high density lipoprotein; VITD, vitamin D; LDL low density lipoprotein; EDUC, level of education } \\
\text { (less than high school, high school, more than high school); DMDMARTL, Marital status (married, } \\
\text { living with partner versus not, single); PAQ180, avg level of physical activity each day. }\end{array}$} \\
\hline
\end{tabular}




\begin{tabular}{|c|c|c|c|c|c|}
\hline $\begin{array}{l}P S A(\mathrm{ng} / \mathrm{ml}) \log 2 \\
\text { transform }\end{array}$ & Q1 & Q2 & Q3 & Q4 & P-value \\
\hline Age(year) & $\begin{array}{l}56.07(12.27) \\
53.00(40.00- \\
85.00)\end{array}$ & $\begin{array}{l}54.83(11.41) \\
52.00(40.00- \\
85.00)\end{array}$ & $\begin{array}{l}59.64(12.27) \\
59.00(40.00- \\
85.00)\end{array}$ & $\begin{array}{l}67.28(11.33) \\
68.00(40.00- \\
85.00)\end{array}$ & $\begin{array}{l}< \\
0.001^{\# \#}\end{array}$ \\
\hline VITD & $\begin{array}{l}58.84(21.21) \\
58.10(9.10- \\
147.00)\end{array}$ & $\begin{array}{l}60.10(20.88) \\
58.70(16.20- \\
232.00)\end{array}$ & $\begin{array}{l}60.90(21.43) \\
60.60(11.80- \\
151.00)\end{array}$ & $\begin{array}{l}60.96(23.23) \\
59.45(9.10- \\
191.00)\end{array}$ & $0.020^{\#}$ \\
\hline $\begin{array}{l}\mathrm{PSA}(\mathrm{ng} / \mathrm{ml}) \log 2 \\
\text { transform }\end{array}$ & $\begin{array}{l}-1.48(0.60) \\
-1.32(-3.84- \\
0.81)\end{array}$ & $\begin{array}{l}-0.44(0.22) \\
-0.45(-0.79- \\
0.06)\end{array}$ & $\begin{array}{l}0.41(0.28) \\
0.40(-0.04- \\
0.91)\end{array}$ & $\begin{array}{l}1.91(0.87) \\
1.68(0.92- \\
6.21)\end{array}$ & $\begin{array}{l}< \\
0.001^{\# \#}\end{array}$ \\
\hline $\begin{array}{l}\text { Zinc (mg) first } \\
\text { day }\end{array}$ & $\begin{array}{l}13.92(12.72) \\
11.75(0.19- \\
264.98)\end{array}$ & $\begin{array}{l}13.75(13.33) \\
11.76(2.32- \\
314.30)\end{array}$ & $\begin{array}{l}12.50(8.08) \\
10.96(0.77- \\
176.77)\end{array}$ & $\begin{array}{l}12.22(11.07) \\
10.24(0.73- \\
279.36)\end{array}$ & $\begin{array}{l}< \\
0.001^{\# \#}\end{array}$ \\
\hline $\begin{array}{l}\text { Hypertension } \\
\text { history }\end{array}$ & & & & & $\begin{array}{l}< \\
0.0001^{\# \#}\end{array}$ \\
\hline 1 & $36.62 \%$ & 34.05 & 38.38 & 46.89 & \\
\hline 2 & 63.38 & 65.95 & 61.62 & 53.11 & \\
\hline Diabetes history & & & & & 0.0048 \\
\hline 1 & 14.32 & 9.65 & 10.78 & 13.15 & \\
\hline 2 & 85.68 & 90.35 & 89.22 & 86.85 & \\
\hline $\begin{array}{l}\text { DMDEDUC2.NEW } \\
\text { NEW }\end{array}$ & & & & & 0.0007 \\
\hline 1 & 7.32 & 7.18 & 7.13 & 10.92 & \\
\hline 2 & 36.45 & 35.30 & 37.13 & 36.99 & \\
\hline 3 & 56.23 & 57.52 & 55.75 & 52.09 & \\
\hline $\begin{array}{l}\text { DMDMARTL.NEW } \\
\text { NEW }\end{array}$ & & & & & $\begin{array}{l}<0.0001 \\
\# \#\end{array}$ \\
\hline 1 & 72.60 & 70.94 & 70.64 & 74.36 & \\
\hline 2 & 21.29 & 22.17 & 24.78 & 23.66 & \\
\hline 3 & 6.11 & 6.89 & 4.58 & 1.98 & \\
\hline $\begin{array}{l}\text { coronary heart } \\
\text { disease }\end{array}$ & & & & & $0.0398^{\#}$ \\
\hline
\end{tabular}

HDL, high density lipoprotein; VITD, vitamin D; LDL low density lipoprotein; EDUC, level of education (less than high school, high school, more than high school); DMDMARTL, Marital status (married, living with partner versus not, single); PAQ180, avg level of physical activity each day. 


\begin{tabular}{|c|c|c|c|c|c|}
\hline $\begin{array}{l}\text { PSA(ng/ml) log2 } \\
\text { transform }\end{array}$ & Q1 & Q2 & Q3 & Q4 & $P$-value \\
\hline 1 & 5.79 & 4.94 & 6.49 & 8.24 & \\
\hline 2 & 94.21 & 95.06 & 93.51 & 91.76 & \\
\hline stroke & & & & & $\begin{array}{l}< \\
0.0001^{\# \#}\end{array}$ \\
\hline 1 & 2.80 & 2.06 & 2.81 & 5.96 & \\
\hline 2 & 97.20 & 97.94 & 97.19 & 94.04 & \\
\hline Race/Ethnicity & & & & & $0.0078^{\# \#}$ \\
\hline $\begin{array}{l}\text { Mexican } \\
\text { American }\end{array}$ & 5.88 & 7.40 & 6.42 & 5.17 & \\
\hline Other Hispanic & 3.44 & 3.71 & 2.55 & 3.21 & \\
\hline $\begin{array}{l}\text { Non-Hispanic } \\
\text { White }\end{array}$ & 76.23 & 76.34 & 74.86 & 77.47 & \\
\hline $\begin{array}{l}\text { Non-Hispanic } \\
\text { Black }\end{array}$ & 9.00 & 8.97 & 10.18 & 10.00 & \\
\hline $\begin{array}{l}\text { Other Race - } \\
\text { Including Multi- } \\
\text { Racial }\end{array}$ & 5.45 & 3.57 & 6.00 & 4.14 & \\
\hline $\begin{array}{l}\text { Smoked at least } \\
100 \text { cigarettes in } \\
\text { life }\end{array}$ & & & & & $0.0148^{\#}$ \\
\hline 1 & 57.61 & 57.77 & 62.07 & 63.48 & \\
\hline 2 & 42.39 & 42.23 & 37.93 & 36.52 & \\
\hline $\begin{array}{l}\text { Enlarged prostate } \\
\text { NEW }\end{array}$ & & & & & $\begin{array}{l}< \\
0.0001^{\# \#}\end{array}$ \\
\hline 1 & 10.62 & 11.33 & 13.28 & 28.11 & \\
\hline 2 & 89.38 & 88.67 & 86.72 & 71.89 & \\
\hline PAQ180 NEW & & & & & $\begin{array}{l}< \\
0.0001^{\# \#}\end{array}$ \\
\hline 1 & 22.67 & 26.27 & 19.25 & 28.44 & \\
\hline 2 & 47.03 & 43.52 & 51.94 & 50.17 & \\
\hline
\end{tabular}

HDL, high density lipoprotein; VITD, vitamin D; LDL low density lipoprotein; EDUC, level of education (less than high school, high school, more than high school); DMDMARTL, Marital status (married, living with partner versus not, single); PAQ180, avg level of physical activity each day. 


\begin{tabular}{|c|c|c|c|c|c|}
\hline $\begin{array}{l}P S A(\mathrm{ng} / \mathrm{ml}) \log 2 \\
\text { transform }\end{array}$ & Q1 & Q2 & Q3 & Q4 & $P$-value \\
\hline 3 & 19.84 & 17.33 & 20.95 & 16.97 & \\
\hline 4 & 10.47 & 12.88 & 7.86 & 4.42 & \\
\hline \multicolumn{6}{|c|}{$\begin{array}{l}\text { HDL, high density lipoprotein; VITD, vitamin D; LDL low density lipoprotein; EDUC, level of education } \\
\text { (less than high school, high school, more than high school); DMDMARTL, Marital status (married, } \\
\text { living with partner versus not, single); PAQ180, avg level of physical activity each day. }\end{array}$} \\
\hline
\end{tabular}

\section{Univariate and multivariate analysis}

Variables that were identified to be significantly associated with serum PSA levels in univariate analysis included lead, cadmium, HDL, age, hypertension history, body mass index, DMDEDUC2, DMDMARTL3, enlarged prostate, coronary heart disease, stroke, PAQ180, smoked at least 100 cigarettes during lifetime, and zinc intake on the first day. The test results for these significant variables were $1.14 \mathrm{ng} / \mathrm{mL}(1.15$, $95 \% \mathrm{Cl}, \mathrm{P}<0.0001), 1.05 \mathrm{ng} / \mathrm{mL}(1.05,95 \% \mathrm{Cl}, \mathrm{P}<0.0001), 0.01(0.01,95 \% \mathrm{Cl}, \mathrm{P}<0.0001), 0.04 \mathrm{ng} / \mathrm{mL}$ (95\% Cl, $\mathrm{P}<0.0001), 0.23$ (95\% Cl, $\mathrm{P}<0.0001), 0.03$ (95\% Cl, $\mathrm{P}<0.0001), 1.03$ (95\% Cl, $\mathrm{P}<0.0001), 0.88$ (95\% Cl, $\mathrm{P}<0.0001), 0.84,(95 \% \mathrm{Cl}, \mathrm{P}<0.0001), 0.8$ (95\% Cl, $\mathrm{P}<0.0001), 0.59$ (95\% Cl, $\mathrm{P}<0.0001), 0.90$ (95\% Cl, $\mathrm{P}<0.0001), 0.28$ (95\% Cl, $\mathrm{P}<0.0001), 0.47$ ( $95 \% \mathrm{Cl}, \mathrm{P}<0.0001), 0.23$ (95\% Cl, $\mathrm{P}=0.0012), 0.44$ $(95 \% \mathrm{Cl}, \mathrm{P}<0.0001), 0.10(95 \% \mathrm{Cl}, \mathrm{P}=0.0047)$, and $0.14(95 \% \mathrm{Cl}, \mathrm{P}<0.0001)$, respectively.

Multivariate analysis indicated that body mass index $(0.02,95 \% \mathrm{Cl}, \mathrm{P}<0.0001)$, DMDMARTL3 $(0.33,95 \%$ $\mathrm{Cl}, \mathrm{P}<0.0001)$, enlarged prostate $(0.59,95 \% \mathrm{Cl}, \mathrm{P}<0.0001)$, and $\mathrm{PAQ} 180(1.54,95 \% \mathrm{Cl}, \mathrm{P}<0.0001)$ were negatively associated with serum PSA levels, whereas lead exposure $(0.08,95 \% \mathrm{Cl}, \mathrm{P}=0.0054)$ and age $(0.03,95 \% \mathrm{Cl}, \mathrm{P}<0.0001)$ were positively associated with serum PSA levels.

The analyses indicated that lead exposure, body mass index, DMDMART (single), enlarged prostate, and age were significantly associated with elevated serum PSA levels, with only lead exposure having a $10 \%$ or more effect size. The results also suggested that cadmium, mercury exposure, or zinc intake was irrelevant to changes in serum PSA levels. 
Table 2

Univariate and multivariate analysis.

\begin{tabular}{|c|c|c|}
\hline Exposure & Univariate & Multivariate \\
\hline Mercury, total (umol/L) log2 transform & $0.01(-0.02,0.03) 0.5889$ & $-0.00(-0.03,0.03) 0.8655$ \\
\hline Lead (umol/L) log2 transform & $0.20(0.16,0.23)<0.0001$ & $0.08(0.03,0.14) 0.0054$ \\
\hline Cadmium (nmol/L) log2 transform & $0.07(0.04,0.10)<0.0001$ & $0.02(-0.02,0.06) 0.4161$ \\
\hline \multicolumn{3}{|l|}{ Diabetes history } \\
\hline 1 & 0 & 0 \\
\hline 2 & $0.02(-0.06,0.11) 0.5932$ & $0.08(-0.04,0.21) 0.2082$ \\
\hline \multicolumn{3}{|l|}{ Hypertension history } \\
\hline 1 & 0 & 0 \\
\hline 2 & $-0.23(-0.30,-0.17)<0.0001$ & $-0.03(-0.12,0.07) 0.5847$ \\
\hline Body mass index,Kg/m2 & $-0.03(-0.03,-0.02)<0.0001$ & $-0.02(-0.03,-0.01)<0.0001$ \\
\hline \multicolumn{3}{|l|}{ DMDEDUC2.NEW NEW } \\
\hline 1 & 0 & 0 \\
\hline 2 & $-0.19(-0.29,-0.10)<0.0001$ & $-0.05(-0.17,0.08) 0.4867$ \\
\hline 3 & $-0.25(-0.34,-0.16)<0.0001$ & $-0.15(-0.28,-0.01) 0.0310$ \\
\hline \multicolumn{3}{|l|}{ DMDMARTL.NEW NEW } \\
\hline 1 & 0 & 0 \\
\hline 2 & $0.07(-0.00,0.15) 0.0601$ & $-0.04(-0.14,0.06) 0.4439$ \\
\hline 3 & $-0.33(-0.48,-0.18)<0.0001$ & $-0.34(-0.54,-0.13) 0.0011$ \\
\hline Alcohol (gm) first day & $-0.00(-0.00,-0.00) 0.0176$ & $-0.00(-0.00,0.00) 0.9762$ \\
\hline $\mathrm{HDL}$ & $0.01(0.01,0.01)<0.0001$ & $0.00(-0.00,0.01) 0.1418$ \\
\hline Poverty income ratio & $-0.02(-0.04,-0.00) 0.0341$ & $0.02(-0.01,0.05) 0.2171$ \\
\hline \multicolumn{3}{|l|}{ Enlarged prostate } \\
\hline 1 & 0 & 0 \\
\hline 2 & $-0.59(-0.68,-0.50)<0.0001$ & $-0.37(-0.49,-0.25)<0.0001$ \\
\hline 7 & $2.97(0.34,5.60) 0.0267$ & 0 \\
\hline 9 & $-0.90(-1.32,-0.48)<0.0001$ & $-0.65(-1.27,-0.03) 0.0398$ \\
\hline LBDLDL & $-0.00(-0.00,-0.00)<0.0001$ & $0.00(-0.00,0.00) 0.4904$ \\
\hline
\end{tabular}




\begin{tabular}{|c|c|c|}
\hline Exposure & Univariate & Multivariate \\
\hline C-reactive protein $(\mathrm{mg} / \mathrm{dL})$ & $0.03(0.00,0.07) 0.0465$ & $0.02(-0.02,0.06) 0.4088$ \\
\hline Glycohemoglobin (\%) & $-0.02(-0.05,0.00) 0.1050$ & $-0.00(-0.04,0.03) 0.8403$ \\
\hline Triglycerides $(\mathrm{mg} / \mathrm{dL})$ & $-0.00(-0.00,-0.00)<0.0001$ & $-0.00(-0.00,-0.00) 0.0032$ \\
\hline \multicolumn{3}{|l|}{ coronary heart disease } \\
\hline 1 & 0 & 0 \\
\hline 2 & $-0.28(-0.40,-0.16)<0.0001$ & $0.01(-0.15,0.16) 0.9480$ \\
\hline \multicolumn{3}{|l|}{ stroke } \\
\hline 1 & 0 & 0 \\
\hline 2 & $-0.47(-0.59,-0.35)<0.0001$ & $-0.15(-0.30,-0.00) 0.0452$ \\
\hline \multicolumn{3}{|l|}{ PAQ180 } \\
\hline 1 & 0 & 0 \\
\hline 2 & $-0.05(-0.16,0.05) 0.3225$ & $0.03(-0.07,0.14) 0.5191$ \\
\hline 3 & $-0.23(-0.37,-0.09) 0.0012$ & $-0.09(-0.22,0.05) 0.2155$ \\
\hline 4 & $-0.44(-0.62,-0.27)<0.0001$ & $-0.06(-0.24,0.12) 0.5022$ \\
\hline 7 & $-0.64(-3.32,2.05) 0.6429$ & $-0.85(-3.36,1.67) 0.5104$ \\
\hline 9 & $-1.54(-2.89,-0.20) 0.0247$ & $-1.84(-3.11,-0.58) 0.0043$ \\
\hline Age,year & $0.04(0.03,0.04)<0.0001$ & $0.03(0.02,0.03)<0.0001$ \\
\hline \multicolumn{3}{|l|}{ Smoked at least 100 cigarettes in life } \\
\hline 1 & 0 & 0 \\
\hline 2 & $-0.10(-0.16,-0.03) 0.0047$ & $0.03(-0.06,0.13) 0.4768$ \\
\hline Zinc (mg) first day log2 transform & $-0.14(-0.17,-0.10)<0.0001$ & $0.00(-0.05,0.05) 0.9102$ \\
\hline VITD & $0.00(0.00,0.00) 0.0180$ & $-0.00(-0.00,0.00) 0.3987$ \\
\hline
\end{tabular}

\section{Adjusted model}

A non-adjusted model and a fully adjusted model were attempted to analyze the associations between serum PSA levels with lead, mercury, cadmium exposure, and zinc intake (Table 3). After adjustment of the variables, including hypertension history, body mass index $\left(\mathrm{kg} / \mathrm{m}^{2}\right)$, diabetes history, DMDEDUC, DMDMARTL, alcohol (gm) first day, HDL, poverty income ratio, enlarged prostate, LBDLDL, C-reactive protein $(\mathrm{mg} / \mathrm{dL})$, glycohemoglobin $(\%)$, triglycerides $(\mathrm{mg} / \mathrm{dL})$, coronary heart disease, stroke, PAQ180, age 
(year), race/ethnicity, smoked at least 100 cigarettes during lifetime, VITD, and zinc (mg) first day, the effect size for lead exposure was reduced by nearly $60 \%$ from 0.196 (multivariate analysis) to 0.079 ( $P=$ 0.00752). The other three variables (mercury exposure, cadmium exposure, and zinc intake) were not significantly associated with changes in serum PSA levels.

\section{GAM}

In the four-subgroup data (Q1-Q4), we observed a non-equidistant change of regression coefficients when linear regression models were used to analyze the relationship between PSA levels and the four variables of interest, i.e., lead exposure, cadmium exposure, mercury exposure, and zinc intake. Therefore, GAM was used to identify the non-linear relationship between these four variables and PSA levels. The advantage of this model is that it allows other variables to be adjusted using a function, which are then included in a regression model. If a non-linear relationship is identified, an inflection point is calculated using a recursive algorithm, followed by an analysis with a weighted linear regression model to integrate data points on both sides of the inflection point. Eventually, a simple linear regression model or a piecewise linear regression model can be established based on the relationship between the logarithmic likelihood ratio with 0.01 selected as a cutoff. In GAM analyses, the variables that had been adjusted included hypertension history, body mass index $\left(\mathrm{kg} / \mathrm{m}^{2}\right)$ (smooth), diabetes history, DMDEDUC, DMDMARTL, alcohol (gm) first day (smooth), HDL (smooth), poverty income ratio, enlarged prostate, LBDLDL (smooth), C-reactive protein (mg/dL) (smooth), glycohemoglobin (\%) (smooth), triglycerides $(\mathrm{mg} / \mathrm{dL})$ (smooth), coronary heart disease, stroke, PAQ180, age (year) (smooth), race/ethnicity, smoked at least 100 cigarettes during lifetime, VITD (smooth), and zinc (mg) first day (smooth). The results showed that only lead exposure was significantly associated with changes in serum PSA levels after the adjustment. Univariate analysis showed a significant association between zinc intake and serum PSA levels, whereas multivariate analysis did not confirm this association; however, when zinc was adjusted in the GAM analysis, a significant association between lead exposure and serum PSA levels was observed. The sum of the evidence indicated that the zinc in blood influences serum PSA levels in an indirect manner, which is also supported by the fact that zinc does not directly interfere with PSA pathways.

As shown in Table 3, the logarithmic likelihood ratios for lead, cadmium, and mercury calculated using the GAM model were $0.168,0.059$, and 0.399 , respectively, which were all greater than the threshold of 0.01 and suggested a piecewise linear regression model. The analysis with the piecewise linear regression model indicated that when the cadmium concentration was less than $6.06 \mu \mathrm{mol} / \mathrm{L}$, an increase of cadmium by a unit led to a slight increase in PSA levels by 0.08 units. Such a small effect is equivalent to a $2 \%$ change compared with the standard used in clinical practice for diagnosis, i.e., PSA at $4 \mathrm{ng} / \mathrm{mL}$. There was no such association when cadmium concentrations were greater than $6.06 \mu \mathrm{mol} / \mathrm{L}$. No association was identified between serum PSA levels and lead or mercury. 
Table 3

Adjusted model and generalized additive model.

\begin{tabular}{|c|c|c|c|}
\hline \multirow[t]{2}{*}{ Exposure } & Non-adjusted model & Fully-adjusted model & GAM model \\
\hline & $(\beta, 95 \% \mathrm{Cl}, \mathrm{P}$ value $)$ & ( $\beta, 95 \% \mathrm{Cl}, \mathrm{P}$ value $)$ & $(\beta, 95 \% \mathrm{Cl}, \mathrm{P}$ value $)$ \\
\hline $\begin{array}{l}\text { Lead (umol/L) log2 } \\
\text { transform }\end{array}$ & $\begin{array}{l}0.196(0.158,0.234)< \\
0.00001\end{array}$ & $\begin{array}{l}0.079(0.021,0.136) \\
0.00752\end{array}$ & $\begin{array}{l}0.070(0.013,0.128) \\
0.01718\end{array}$ \\
\hline Q1(-6.15 to -3.86$)$ & 0 & 0 & 0 \\
\hline Q2 (-3.84 to -3.32$)$ & $\begin{array}{l}0.278(0.186,0.371)< \\
0.00001\end{array}$ & $\begin{array}{l}0.164(0.035,0.293) \\
0.01258\end{array}$ & $\begin{array}{l}0.143(0.014,0.272) \\
0.02969\end{array}$ \\
\hline Q3 (-3.31 to -2.75$)$ & $\begin{array}{l}0.321(0.229,0.413)< \\
0.00001\end{array}$ & $\begin{array}{l}0.152(0.024,0.281) \\
0.02014\end{array}$ & $\begin{array}{l}0.132(0.003,0.260) \\
0.04431\end{array}$ \\
\hline Q4 (-2.74 to -0.67$)$ & $\begin{array}{l}0.431(0.339,0.523)< \\
0.00001\end{array}$ & $\begin{array}{l}0.142(0.009,0.275) \\
0.03679\end{array}$ & $\begin{array}{l}0.115(-0.020,0.249) \\
0.09426\end{array}$ \\
\hline$P$ for trend & $\begin{array}{l}0.133(0.104,0.162)< \\
0.00001\end{array}$ & $\begin{array}{l}0.037(-0.005,0.079) \\
0.08412\end{array}$ & $\begin{array}{l}0.029(-0.013,0.072) \\
0.17701\end{array}$ \\
\hline $\begin{array}{l}\text { Cadmium (nmol/L) log2 } \\
\text { transform }\end{array}$ & $\begin{array}{l}0.073(0.045,0.101)< \\
0.00001\end{array}$ & $\begin{array}{l}0.035(-0.005,0.075) \\
0.08658\end{array}$ & $\begin{array}{l}0.027(-0.014,0.067) \\
0.19236\end{array}$ \\
\hline Q1 (-0.17 to 0.97) & 0 & 0 & 0 \\
\hline Q2 (1.03 to 1.72$)$ & $\begin{array}{l}0.234(0.141,0.326)< \\
0.00001\end{array}$ & $\begin{array}{l}0.040(-0.086,0.166) \\
0.53269\end{array}$ & $\begin{array}{l}0.031(-0.094,0.156) \\
0.62969\end{array}$ \\
\hline Q3 (1.76 to 2.62 ) & $\begin{array}{l}0.404(0.312,0.496)< \\
0.00001\end{array}$ & $\begin{array}{l}0.165(0.039,0.290) \\
0.01035\end{array}$ & $\begin{array}{l}0.145(0.020,0.270) \\
0.02351\end{array}$ \\
\hline Q4 (2.63 to 6.29 ) & $\begin{array}{l}0.283(0.191,0.376)< \\
0.00001\end{array}$ & $\begin{array}{l}0.124(-0.007,0.255) \\
0.06269\end{array}$ & $\begin{array}{l}0.092(-0.040,0.224) \\
0.17315\end{array}$ \\
\hline$P$ for trend & $<0.00001$ & 0.02186 & 0.07494 \\
\hline $\begin{array}{l}\text { Mercury, total (umol/L) } \\
\text { log2 transform }\end{array}$ & $\begin{array}{l}0.006(-0.016,0.028) \\
0.58892\end{array}$ & $\begin{array}{l}-0.010(-0.040 \\
0.021) 0.53175\end{array}$ & $\begin{array}{l}-0.008(-0.039,0.023) \\
0.60519\end{array}$ \\
\hline
\end{tabular}

Non-adjusted model adjusts for: None

Fully-adjusted model adjust for: Hypertension history, body mass index $\left(\mathrm{kg} / \mathrm{m}^{2}\right)$, diabetes history, DMDEDUC, DMDMARTL, alcohol (gm) first day, HDL, poverty income ratio, enlarged prostate, LBDLDL, C-reactive protein $(\mathrm{mg} / \mathrm{dL})$, glycohemoglobin $(\%)$, triglycerides $(\mathrm{mg} / \mathrm{dL})$, coronary heart disease, stroke, PAQ180, age (year), race/ethnicity, smoked at least 100 cigarettes during lifetime, VITD, zinc (mg) first day.

GAM model was adjusted for: Hypertension history, body mass index $\left(\mathrm{kg} / \mathrm{m}^{2}\right)$ (smooth), diabetes history, DMDEDUC2.NEW NEW, DMDMARTL.NEW NEW, alcohol (gm) first day (smooth), HDL (smooth), poverty income ratio, enlarged prostate, LBDLDL (Smooth), C-reactive protein $(\mathrm{mg} / \mathrm{dL}$ ) (smooth), glycohemoglobin (\%) (smooth), triglycerides (mg/dL) (smooth), coronary heart disease, stroke, PAQ180, age (year) (smooth), race/ethnicity, smoked at least 100 cigarettes during lifetime, VITD (smooth), zinc (mg) first day (smooth). Generalized additive models were applied. 


\begin{tabular}{|c|c|c|c|}
\hline \multirow[t]{2}{*}{ Exposure } & Non-adjusted model & Fully-adjusted model & GAM model \\
\hline & $(\beta, 95 \% \mathrm{Cl}, \mathrm{P}$ value $)$ & $(\beta, 95 \% \mathrm{Cl}, \mathrm{P}$ value $)$ & $(\beta, 95 \% \mathrm{Cl}, \mathrm{P}$ value $)$ \\
\hline Q1 (-1.03 to 1.29) & 0 & 0 & 0 \\
\hline Q2 (1.32-2.30) & $\begin{array}{l}-0.032(-0.126,0.062) \\
0.50588\end{array}$ & $\begin{array}{l}-0.000(-0.124 \\
0.124) 0.99601\end{array}$ & $\begin{array}{l}-0.001(-0.125,0.122) \\
0.98525\end{array}$ \\
\hline Q3(2.31 to 3.31$)$ & $\begin{array}{l}0.014(-0.080,0.109) \\
0.76542\end{array}$ & $\begin{array}{l}-0.021(-0.146 \\
0.105) 0.74448\end{array}$ & $\begin{array}{l}-0.019(-0.144,0.106) \\
0.76646\end{array}$ \\
\hline Q4 (3.32 to 8.74$)$ & $\begin{array}{l}0.028(-0.067,0.122) \\
0.56739\end{array}$ & $\begin{array}{l}-0.038(-0.169 \\
0.092) 0.56309\end{array}$ & $\begin{array}{l}-0.043(-0.173,0.086) \\
0.51268\end{array}$ \\
\hline$P$ for trend & 0.36689 & 0.50872 & 0.47009 \\
\hline $\begin{array}{l}\text { Zinc }(\mathrm{mg}) \text { first day log2 } \\
\text { transform }\end{array}$ & $\begin{array}{l}-0.136(-0.174,-0.097) \\
<0.00001\end{array}$ & $\begin{array}{l}-0.016(-0.090 \\
0.058) 0.67581\end{array}$ & $\begin{array}{l}0.042(-0.154,0.239) \\
0.67255\end{array}$ \\
\hline low & 0 & 0 & 0 \\
\hline medium & $\begin{array}{l}-0.139(-0.221,-0.058) \\
0.00079\end{array}$ & $\begin{array}{l}-0.071(-0.178 \\
0.035) 0.19049\end{array}$ & $\begin{array}{l}-0.091(-0.205,0.023) \\
0.11889\end{array}$ \\
\hline high & $\begin{array}{l}-0.325(-0.407,-0.244) \\
<0.00001\end{array}$ & $\begin{array}{l}-0.112(-0.239 \\
0.015) 0.08366\end{array}$ & $\begin{array}{l}-0.176(-0.340 \\
-0.013) 0.03465\end{array}$ \\
\hline$P$ for trend & $<0.00001$ & 0.07877 & 0.08324 \\
\hline \multicolumn{4}{|c|}{ Non-adjusted model adjusts for: None } \\
\hline \multicolumn{4}{|c|}{$\begin{array}{l}\text { Fully-adjusted model adjust for: Hypertension history, body mass index }\left(\mathrm{kg} / \mathrm{m}^{2}\right) \text {, diabetes history, } \\
\text { DMDEDUC, DMDMARTL, alcohol (gm) first day, } \mathrm{HDL} \text {, poverty income ratio, enlarged prostate, LBDLDL, } \\
\text { C-reactive protein (mg/dL), glycohemoglobin ( } \%) \text {, triglycerides (mg/dL), coronary heart disease, stroke, } \\
\text { PAQ180, age (year), race/ethnicity, smoked at least } 100 \text { cigarettes during lifetime, VITD, zinc (mg) first } \\
\text { day. }\end{array}$} \\
\hline \multicolumn{4}{|c|}{$\begin{array}{l}\text { GAM model was adjusted for: Hypertension history, body mass index }\left(\mathrm{kg} / \mathrm{m}^{2}\right) \text { (smooth), diabetes } \\
\text { history, DMDEDUC2.NEW NEW, DMDMARTL.NEW NEW, alcohol (gm) first day (smooth), HDL } \\
\text { (smooth), poverty income ratio, enlarged prostate, LBDLDL (Smooth), C-reactive protein (mg/dL) } \\
\text { (smooth), glycohemoglobin (\%) (smooth), triglycerides (mg/dL) (smooth), coronary heart disease, } \\
\text { stroke, PAQ180, age (year) (smooth), race/ethnicity, smoked at least } 100 \text { cigarettes during lifetime, } \\
\text { VITD (smooth), zinc (mg) first day (smooth). Generalized additive models were applied. }\end{array}$} \\
\hline
\end{tabular}

\section{Subgroup analysis}

Univariate analyses showed that lead, cadmium, and zinc were all significantly associated with changes in serum PSA levels. Multivariate analyses, using a fully adjusted model, and GAM analysis only detected an association between lead and serum PSA. Nevertheless, when zinc was treated as a variable being adjusted in multivariate analysis, the fully adjusted model, or GAM, associations between serum PSA and lead or cadmium could be detected. Thus, we divided the sample into three strata (T1: 0.19-8.69 (low), T2: 8.75-14.03 (median), and T3: 14.12-315.17 (high)) based on zinc intake, and analyzed each of these three strata separately using various models including a non-adjusted model, an adjusted model I, 
and an adjusted model II (Table 4). The results showed that only in the T3 (high level of zinc intake) stratum, exposure to cadmium or lead could increase serum PSA levels: an increase of cadmium by 1 unit led to an increase in PSA by 1.06 unit, while an increase in lead by 1 unit yielded an increase in PSA by 1.09 units. Such effects are equivalent to $25 \%$ of changes compared with the standard for diagnosis, which is quite significant in clinical practice. No such associations were detected in the T1 (low level of zinc intake) or T2 (median level of zinc intake) strata. These results suggest that increased zinc intake may interfere with serum cadmium to increase serum PSA levels in a synergistic manner.

Table 4

Subgroup analysis.

\begin{tabular}{|llll|}
\hline Exposure & Non-adjusted model & Adjust model I & Adjust model II \\
\hline \begin{tabular}{llll|} 
Zinc $(\mathrm{mg})$ first day log2 \\
transform
\end{tabular} & $\begin{array}{l}-0.136(-0.174,-0.097) \\
<0.00001\end{array}$ & $-0.016(-0.090,0.058)$ & $\begin{array}{l}0.042(-0.154,0.239) \\
0.67255\end{array}$ \\
\hline $\begin{array}{l}\text { Zinc }(\mathrm{mg}) \text { first day log2 } \\
\text { transform }\end{array}$ & & & \\
\hline T1 & 0 & 0 & 0 \\
\hline T2 & $-0.139(-0.221,-0.058)$ & $-0.071(-0.178,0.035)$ & $-0.091(-0.205,0.023)$ \\
& 0.00079 & 0.19049 & 0.11889 \\
\hline T3 & $-0.325(-0.407,-0.244)$ & $-0.112(-0.239,0.015)$ & $-0.176(-0.340,-0.013)$ \\
& $<0.00001$ & 0.08366 & 0.03465 \\
\hline P for trend & $<0.00001$ & 0.07877 & 0.08324 \\
\hline Non-adjusted model adjusts for: none. & & \\
\hline
\end{tabular}

Adjusted model I adjusts for: demography univariate.

Adjusted model II adjusts for: all univariate in Table I. 
Table 5

Interaction relationships.

\begin{tabular}{|lllll|}
\hline Model & $\begin{array}{l}\text { DR1TZINC.T3: } \\
\text { LOW }\end{array}$ & $\begin{array}{l}\text { DR1TZINC.T3: } \\
\text { MEDIUM }\end{array}$ & $\begin{array}{l}\text { DR1TZINC.T3: } \\
\text { HIGH }\end{array}$ & $\begin{array}{l}\text { P } \\
\text { interaction }\end{array}$ \\
\hline $\begin{array}{l}\text { Mercury, total (umol/L) } \\
\text { log2 transform }\end{array}$ & & & & \\
\hline Model & $-0.01(-0.06$, & $0.01(-0.05,0.06)$ & $-0.04(-0.09,0.02)$ & 0.4921 \\
& $0.04) 0.6586$ & 0.7373 & 0.1873 & \\
\hline $\begin{array}{l}\text { Lead (umol/L) log2 } \\
\text { transform }\end{array}$ & & & \\
\hline Model II* & $0.06(-0.04,0.16)$ & $0.04(-0.06,0.15)$ & $0.12(0.03,0.22)$ & 0.5056 \\
& 0.2390 & 0.4020 & 0.0122 & \\
\hline Cadmium (nmol/L) log2 & & & & \\
transform & & & & 0.0249 \\
\hline Model II* & $-0.03(-0.10$, & $0.07(-0.01,0.14)$ & $0.08(0.01,0.14)$ & \\
& $0.03) 0.3253$ & 0.0748 & 0.0268 & \\
\hline
\end{tabular}

\section{Discussion}

The purpose of this study was to evaluate the effects of lead, cadmium, and mercury exposure, as well as zinc intake on serum PSA levels in PCa-free men. Early studies have shown that exposure to lead, cadmium, and mercury poses a significant threat to human health. Zinc is considered to be a useful metal for biological growth and disease recovery, although it does have potential side effects. This study showed that lead exposure increased serum PSA levels in normal men, whereas cadmium or mercury exposure or zinc intake did not affect serum PSA levels. Interestingly, we found that when zinc intake was $14.12 \mathrm{mg} /$ day or higher (high dose), exposure to cadmium significantly increased serum PSA levels ( $\beta=$ $1.06,95 \% \mathrm{Cl}$ ), indicating a possible synergistic effect between zinc and cadmium on serum PSA.

Toxicological and physiological studies have shown that lead, cadmium, mercury, and zinc are toxic heavy metals, which can cause various degrees of damage to the human body. Lead may aggregate in blood, liver, and kidney, which can lead to serious toxicity to the nervous system, kidney, and testis [41]. A study has shown that synergism between lead, cadmium, and mercury can cause damage to kidney function [42]. Lead also targets testicular Sertoli cells, which may affect sperm production [43]. Lead toxicity in a children's nervous system will cause typical neurological symptoms [44]. Long time exposure to lead increases the risk of Alzheimer's disease in the elderly [45]. Acute cadmium poisoning usually occurs 12-24 hours after exposure, with severe respiratory symptoms and necrosis of the liver and kidney, while chronic cadmium poisoning is mainly characterized by toxicity in the cardiovascular, cerebrovascular, kidney, and digestive tract [46-49]. Mercury volatilizes easily and chronic exposure to mercury causes nervous system damage and visual impairment, which poses a high level of risk in certain occupations [50-53]. 
It has been a topic of debate as to whether zinc is beneficial in human health. For example, zinc has long been documented to be beneficial in epilepsy, which may be traced back to a study in 1786 [54]. Studies have also shown that zinc may assist in wound healing, and zinc has been applied in the treatment of male infertility [57], impotence [58] and chronic prostatitis [59]. Recent molecular and cellular studies indicated that reduced zinc concentrations were associated with induction or aggravation of tumorigenesis [60-62]. Ogawa et al. found that skin disorders were associated with zinc deficiency [55]. However, other studies have claimed that long term intake of high doses of zinc may cause serious toxicity in the liver and kidney [63-64] and may negatively affect the immune system [65]. Nevertheless, very few epidemiological studies with limited sample sizes have been conducted to test whether these metals have an effect on prostate glands.

Heavy metals aggregate in target organs to cause damage; therefore, investigation of the accumulation of each of the four metals in the prostate may help us understand the relationship between serum PSA levels and these heavy metals. Lead exposure has been reported to be associated with an increased risk of PCa or benign prostatic hyperplasia [66], which may be a trigger for PSA elevation. A study based on only 57 subjects showed no association between serum lead and PSA levels [67], but there was no evidence for the accumulation of lead in the prostate gland.

Like the liver and kidney, the prostate is considered a target organ of cadmium deposition [68]. International cancer research institutes and epidemiological studies have shown that cadmium exposure may be a risk factor for both prostate and renal cancer [69-70]. An in vitro study also showed that cadmium can induce malignant prostate tumors [71]. However, our results based on previous data did not show such a relationship between cadmium exposure and PSA levels, which might have been because of: (1) a potential bias due to the small sample sizes in each of these studies, and (2) suboptimal timing for data collection in these studies. Interestingly, our results indicated that cadmium exposure increased serum PSA levels when zinc intake exceeded $14.12 \mathrm{mg} /$ day.

Based on NHANES data of 1,320 male cases over 40 years old, Edwin et al. found that dietary zinc intake less than $12.67 \mathrm{mg} /$ day was associated with a protective effect on injured prostate glands, while such an association was not observed in the cohort with a higher zinc intake [72], which was partly consistent with our conclusion. Terrence et al. analyzed NHANES data for 6,488 cases with cadmium exposure and found a significant association between zinc intake and serum cadmium [73], which also reiterates what we found. In our study, where the average zinc intake among participants was $13.10 \mathrm{mg} /$ day, we found that $14.12 \mathrm{mg} /$ day was the critical value for zinc intake, beyond which cadmium exposure significantly increased serum PSA levels. Of note is that this critical value was lower than the World Health Organization's suggested standard of $15 \mathrm{mg} /$ day but higher than the dose of $12.67 \mathrm{mg} /$ day recommended by Edwin et al. [72]. An interesting question would be whether men living in cadmiumexposed areas should follow the World Health Organization's recommended zinc intake dose (15 $\mathrm{mg} /$ day), which may artificially increase serum PSA levels leading to a false PCa diagnosis. The vast majority of research on the effect of zinc intake suggests that zinc might protect against PCa; nevertheless, a study based on 2,000 Spaniards indicated that high levels of zinc intake will increase the 
risk of PCa [34]. The sum of the evidence seems to support our findings that zinc and cadmium might increase serum PSA levels in a synergistic manner, which is similar to the conclusions reached by Mahmoud et al. [75].

This study had a number of limitations. First, there was the potential for selection bias and inaccurate data, which was based on participants' memory, and this may have made it difficult to identify associations between serum PSA levels and the metals studied. Second, only US men were included in the study, which may limit the generalizability of the results. Third, there were no other clinical data for determining whether the increase in PSA was because of physiological or pathological causes. Last, the zinc intake data were completely based on participants' recall of the previous 24 hours, thus preventing long-term and sustainable follow-up.

However, compared with previous studies, our research had the following advantages. First, this study included a total of 5,089 participants from NHANES, which may have reduced the potential biases because of small sample sizes. Second, we used advanced methodologies, including the GAM model, to detect and analyze the potential non-linear associations between serum PSA levels and these metals. In addition, the analysis of subgroups was used to identify the complex relationship between serum PSA levels and the metals with various zinc intake doses.

\section{Conclusions}

We found that cadmium exposure increased serum PSA levels given that zinc intake exceeded 14.12 $\mathrm{mg} /$ day, leading to a potential overdiagnosis of PCa. Thus, local standards for PSA screening need to be established, and different zinc supplementation strategies could be developed for geographical areas with different levels of cadmium exposure. Our study also indicated that risk factors for PCa should be evaluated with environmental factors taken into account. The underlying biochemical mechanisms involved in the potential synergetic effect of zinc and cadmium on serum PSA levels warrant further investigation.

\section{Abbreviations}

PSA:Serum prostate-specific antigen; PCa:Prostate cancer; USCS:United States Cancer Statistics; UCDCP: US Centers for Disease Control and Prevention; FDA: US Food and Drug Administration; NHANES:National Health and Nutrition Examination Survey; ALT:alanine aminotransferase; BUN:serum urea nitrogen.

\section{Declarations}

\section{Consent for publication}

Not applicable. 


\section{Acknowledgements}

This research did not receive any specifc grant from funding agencies in the public, commercial, or notfor-proft sectors. The present authors would like to express their gratitude to Guizhou provincial people's hospital for funding this research. We thank Ejear, Ph.D., for editing the English text of a draft of this manuscript.

\section{Author contributions}

Zhu. J.G, Chen. C and Chen. W. M designed the research. Chen. X. Y and Song. J. K performed the research. Yuan. D. B, Zhu. G. H and Liu . H analyzed the data. Chen. W. M, Chen. X. Y and Hu. B wrote the paper. Zhu. J. G and Sun. F review, editing and funding the paper. All authors read and approved the final manuscript.

\section{Fundings:}

This study was supported by The Science and Technology Plan Project of Guiyang in 2019 [2019]2-15, The High-level innovative talent project of Guizhou Province in 2018 [2018]5639.

\section{Data Availability Statement}

All data can be obtained by the corresponding author.

Ethics approval and consent to participateNot applicable.

Declaration of Competing Interests.

1 To the best of our knowledge, the named authors have no conflict of interest, financial or otherwise.

2 No support, financial or otherwise, has been received from any organization that may have an interest in the submitted work, and no relationships or activities that could appear to have influenced the submitted work.

3 Informed consent has been obtained from all patients by NIH included in this study.

\section{References}

1. Singh SD, Henley SJ, Ryerson AB. Surveillance for Cancer Incidence and Mortality - United States, 2012. MMWR Morb Mortal Wkly Rep. 2016;63:17-58.

2. Siegel RL, Miller KD, Jemal A. Cancer statistics, 2018. Cancer J Clin. 2018;68:7-30.

3. Kimberly D, Leticia N, Angela B, et al. Cancer treatment and survivorship statistics, CA Cancer J Clin. 2019 Jun 11. doi:10.3322/caac.21565. 
4. Ferlay J, Shin H, Bray F, et al. GLOBOCAN 2008, Cancer incidence and mortality worldwide: IARC CancerBase No.10.Lyon, France. International Agency for Research on Cancer; 2010.

5. Center MM, Jemal A, Lortet Tieulent J, Ward E, Ferlay J, Brawley O, et al. International variation in prostate cancer incidence and mortality rates. Eur Urol. 2012;61:1079 1092.

6. Whittemore AS, Kolonel LN, Wu AH, John EM, Gallagher RP, Howe GR, et al. Prostate cancer in relation to diet, physical activity, and body size in blacks, whites, and Asians in the United States and Canada. J Natl Cancer Inst. 1995;87:652-61.

7. Shimizu H, Ross RK, Bernstein L, Yatani R, Henderson BE, Mack TM. Cancers of the prostate and breast among Japanese and white immigrants in Los Angeles County. Br J Cancer. 1991;63:963-6.

8. Kuriyama M, Wang MC, Papsidero LD, et al. Quantitation of prostate specific antigen in serum by a sensitive enzyme immunoassay. Cancer Res. 1980;40:4658-62.

9. Kuriyama $\mathrm{M}$, Wang $\mathrm{MC}$, Lee $\mathrm{Cl}$, et al. Use of human prostate-specific antigen in monitoring prostate cancer. Cancer Res. 1981 Oct;41(10):3874-6.

10. Shabsigh A. To Treat or Not to Treat. Journal of Men's Health. 2019;15(1):e10-1.

11. Wang A, Lazo M, Carter H,et al. Association Between Liver Fibrosis and Serum Prostate Specific Antigen (PSA) Among US Men: National Health and Nutrition Examination Survey (NHANES) 20012010. Cancer Epidemiol Biomarkers Prev. 2019 Jun 3. pii: cebp.0145.2019. doi: 10.1158/1055-9965.

12. Gao X, Bao T, Yang H, et al. The association of metabolic syndrome and its components with serum prostate-specific antigen levels. Eur J Cancer Prev. 2019 Mar 25.

doi:10.1097/CEJ.0000000000000508.

13. Klaassen Z, Wallis C, Goldberg H, et al. The association between physician trust and prostate-specific antigen screening: Implications for shared decision-making. Can Urol Assoc J. 2018 Jun 19. doi:10.5489/cuaj.5351.

14. Khosravi A, Nemati E, Soleimanian M, et al. Association between prostate specific antigen levels and coronary artery angioplasty. J Renal Inj Prev. 2016 Dec 15;6(2):132-136. doi: 10.15171/jrip.

15. Klaassen Z, Howard L, Moreira D, et al. Association of Obesity-Related Hemodilution of ProstateSpecific Antigen, Dihydrotestosterone, and Testosterone. Prostate. 2017 Apr;77(5):466-70. doi:10.1002/pros.23285.

16. Shirazi M, Ariafar A, Zeyghami S, et al. Association of diet with prostate specific antigen and prostate volume. Nephrourol Mon. 2014 Jul 5;6(4): e19411. doi: 10.5812/numonthly.19411.

17. Tikkinen $K$, Dahm P, Lytvyn L, et al. Prostate cancer screening with prostate-specific antigen (PSA) test: a clinical practice guideline. BMJ. 2018 Sep 5;362:k3581. doi: 10.1136/bmj.k3581.

18. utz PM, Wilson TJ, Ireland $\mathrm{J}$, et al. Elevated immunoglobulin E levels in children with exposure to environmental lead. Toxicology. 1999;134:63-78.

19. Wittman R, Hu H. Cadmium exposure and nephropathy in a 28 years-old female metals worker. Enviro Health Perspect. 2002;110(12):1261-6. 
20. National Center for Environmental Health. Third National Report on Human Exposure to Environmental Chemicals. Atllanta, GA: National Center for Environmental Health. Division of Laboratory Science; Centers for Disease Control and Prevention, Department of Health and Human Service; 2005. pp. 25-30. Report nr NCEH pub. N0.05-0570.

21. Sahmoun A, Case L, Jackson S, et al. Cadmium and prostate cancer: a article epidemiologic analysis. Cancer Invest. 2005;23(3):256-63.

22. Rapisarda V, Miozzi E, Loreto $C$, et al. Cadmium exposure and prostate cancer: insights, mechanisms and perspectives. Front Biosci (Landmark Ed). 2018 Mar 1; 23:1687-1700.

23. Wu CC1, Pu YS, Wu HC, et al. Reversed association between levels of prostate specific antigen and levels of blood cadmium and urinary cadmium. Chemosphere. 2011 May;83(8):1188-91. doi: 10.1016/j.chemosphere.

24. Burger J, Mizrahi D, Tsipoura N, et al. Mercury, Lead, Cadmium, Cobalt, Arsenic and Selenium in the Blood of Semipalmated Sandpipers (Calidris pusilla) from Suriname, South America: Age-related Differences in Wintering Site and Comparisons with a Stopover Site in New Jersey, USA. Toxics. 2018 May 9;6(2). pii: E27. doi: 10.3390/toxics6020027.

25. Zefferino R, Piccoli C, Ricciardi N, et al. Possible Mechanisms of Mercury Toxicity and Cancer Promotion: Involvement of Gap Junction Intercellular Communications and Inflammatory Cytokines. Oxid Med Cell Longev. 2017; 2017:7028583. doi: 10.1155/2017/7028583.

26. John LA, Case. Shewing the Efficacy of Flowers of Zinc, in the Epilepsy. Lond Med J. 1786; 7(Pt 1): 52-54.

27. Ogawa Y, Kawamura T, Shimada S. Zinc and skin biology. Arch Biochem Biophys. 2016 Dec 1; 611:113-119.

28. Mirastschijski U, Martin A, Jorgensen LN, et al. Zinc, copper, and selenium tissue levels and their relation to subcutaneous abscess, minor surgery, and wound healing in humans. Biol Trace Elem Res. 2013 Jun;153(1-3):76-83.

29. Chu DS. Zinc. A small molecule with a big impact on sperm function. PLoS Biol. 2018 Jun 7;16(6): e2006204.

30. Kerns K, Zigo M, Sutovsky P. Zinc. A Necessary Ion for Mammalian Sperm Fertilization Competency. Int J Mol Sci. 2018 Dec 18;19(12). pii: E4097.

31. Goodarzi D, Cyrus A, Baghinia MR, et al. The efficacy of zinc for treatment of chronic prostatitis. Acta Med Indones. 2013 Oct;45(4):259-64.

32. Costello L, Franklin R. Decreased zinc in the development and progression of malignancy: an important common relationship and potential for prevention and treatment of carcinomas. Expert Opin Ther Targets. 2017 Jan;21(1):51-66.

33. Costello L, Franklin R. A comprehensive review of the role of zinc in normal prostate function and metabolism; and its implications in prostate cancer. Arch Biochem Biophys. 2016 Dec 1; 611:100112. 
34. Costello LC, Zou J, Franklin RB. In situ clinical evidence that zinc levels are decreased in breast invasive ductal carcinoma. Cancer Causes Control. 2016 Jun;27(6):729-35.

35. Gutiérrez-González E, Castelló A, Fernández-Navarro P, et al. Dietary Zinc and Risk of Prostate Cancer in Spain: MCC-Spain Study. Nutrients. 2018 Dec 20;11(1). pii: E18.

36. Zhao J, Wu Q, Hu X, et al. Comparative study of serum zinc concentrations in benign and malignant prostate disease: A Systematic Review and Meta-Analysis. Sci Rep. 2016 May;12:6:25778.

37. Adolfsson PI, Bloth B, Hägg S, et al. Zinc induces a bell-shaped proliferative dose-response effect in cultured smooth muscle cells from benign prostatic hyperplasia. Urology. 2015 Mar;85(3):704.e15-9.

38. Pizent A, Colak B, Kljaković Z, et al. Prostate-specific antigen (PSA) in serum in relation to blood lead concentration and alcohol consumption in men. Arh Hig Rada Toksikol. 2009 Mar;60(1):69-78. doi:10.2478/10004-1254-60-2009-1901.

39. van Wijngaarden E, Singer E, Palapattu G. Prostate-specific antigen levels in relation to cadmium exposure and zinc intake: results from the 2001-2002 National Health and Nutrition Examination Survey. Prostate. 2008 Feb 1;68(2):122-8.

40. Liu S, Wang X, Lu Y, et al. The effects of intraoperative cryoprecipitate transfusion on acute renal failure following orthotropic liver transplantation. Hepatol Int. 2013;7:901-10.

41. Andjelkovic M, Buha Djordjevic A, Antonijevic E, et al. Toxic Effect of Acute Cadmium and Lead Exposure in Rat Blood, Liver, and Kidney. Int J Environ Res Public Health. 2019 Jan 18;16(2). pii: E274. doi: 10.3390/ijerph16020274.

42. Jain R. Synergistic impact of co-exposures to toxic metals cadmium, lead, and mercury along with perfluoroalkyl substances on the healthy kidney function. Environ Res. 2019 Feb; 169:342-347. doi: 10.1016/j.envres.2018.11.037.

43. Ramos-Treviño J, Bassol-Mayagoitia S, Hernández-Ibarra J, et al. Toxic Effect of Cadmium, Lead, and Arsenic on the Sertoli Cell: Mechanisms of Damage Involved. DNA Cell Biol. 2018 Jul;37(7):600-608. doi: 10.1089/dna.2017.4081. Epub 2018 May 10.

44. Marissa H, Bryan S, Alan D. Children with Autism Spectrum Disorder and Lead Poisoning: Diagnostic Challenges and Management Complexities. Clin Pediatr (Phila). 2019 Jun;58(6):605-612. doi: $10.1177 / 0009922819839237$.

45. Xu L, Zhang W, Liu X, et al. Circulatory Levels of Toxic Metals (Aluminum, Cadmium, Mercury, Lead) in Patients with Alzheimer's Disease: A Quantitative Meta-Analysis and Systematic Review. J Alzheimers Dis. 2018;62(1):361-72. doi:10.3233/JAD-170811.

46. Andujar P, Bensefa-Colas L, Descatha A. Acute and chronic cadmium poisoning. Rev Med Interne. 2010 Feb;31(2):107-15. doi:10.1016/j.revmed.2009.02.029.

47. Johri N, Jacquillet G, Unwin R. Heavy metal poisoning: the effects of cadmium on the kidney. Biometals. 2010 Oct;23(5):783-92. doi:10.1007/s10534-010-9328-y.

48. Zhang X, Xiao X, Li Y, et al. Analysis of clinical features of mild chronic cadmium poisoning induced by different causes.Zhonghua Lao Dong Wei Sheng Zhi Ye Bing Za Zhi. 2013 Oct;31(10):763-5. 
49. Nai G, Filho M, Estrella M, et al. Study of the influence of the ph of water in the initiation of digestive tract injury in cadmium poisoning in rats. Toxicol Rep. 2015 Jul 19; 2:1033-1038. doi: 10.1016/j.toxrep.2015.07.012.

50. Li L, Jiang B, Lai J, et al. Change in peripheral nervous conduction velocity in patients with occupational chronic mercury poisoning and related influencing factors. Zhonghua Lao Dong Wei Sheng Zhi Ye Bing Za Zhi. 2017 Aug 20;35(8):598-602. doi: 10.3760/cma.j.issn.1001-9391.

51. Prantsidis A, Raikos N, Pantelakis I, et al. Unusual mercury poisoning from tattoo dye.Hippokratia. 2017 Oct-Dec;21(4):197-200.

52. Do S, Lee C, Kim J, et al. Cases of acute mercury poisoning by mercury vapor exposure during the demolition of a fluorescent lamp factory. Ann Occup Environ Med. 2017 Jun 20; 29:19. doi: 10.1186/s40557-017-0184-x. eCollection 2017.

53. Smiechowicz J, Skoczynska A, Nieckula-Szwarc A, et al. Occupational mercury vapour poisoning with a respiratory failure, pneumomediastinum and severe quadriparesis. SAGE Open Med Case Rep. 2017 Feb 23; 5:2050313X17695472. doi: 10.1177/2050313X17695472. eCollection 2017.

54. John LA, Case. Shewing the Efficacy of Flowers of Zinc, in the Epilepsy. Lond Med J. 1786; 7(Pt 1): 52-54.

55. Ogawa Y, Kawamura T, Shimada S. Zinc and skin biology. Arch Biochem Biophys. 2016 Dec 1; 611:113-119.

56. Mirastschijski U, Martin A, Jorgensen LN, et al. Zinc, copper, and selenium tissue levels and their relation to subcutaneous abscess, minor surgery, and wound healing in humans. Biol Trace Elem Res. 2013 Jun;153(1-3):76-83.

57. Chu DS. Zinc. A small molecule with a big impact on sperm function. PLoS Biol. 2018 Jun 7;16(6): e2006204.

58. Kerns K, Zigo M, Sutovsky P. Zinc. A Necessary Ion for Mammalian Sperm Fertilization Competency. Int J Mol Sci. 2018 Dec 18;19(12). pii: E4097.

59. Goodarzi D, Cyrus A, Baghinia MR, et al. The efficacy of zinc for treatment of chronic prostatitis. Acta Med Indones. 2013 Oct;45(4):259-64.

60. Costello LC, Franklin RB. Decreased zinc in the development and progression of malignancy: an important common relationship and potential for prevention and treatment of carcinomas. Expert Opin Ther Targets. 2017 Jan;21(1):51-66.

61. Costello LC, Franklin RB. A comprehensive review of the role of zinc in normal prostate function and metabolism; and its implications in prostate cancer. Arch Biochem Biophys. 2016 Dec 1; 611:100112.

62. Costello LC, Zou J, Franklin RB. In situ clinical evidence that zinc levels are decreased in breast invasive ductal carcinoma. Cancer Causes Control. 2016 Jun;27(6):729-35.

63. Bozym R, Chimienti F, Giblin L, et al. Free zinc ions outside a narrow concentration range are toxic to a variety of cells in vitro. Exp Biol Med (Maywood). 2010 Jun;235(6):741-50.

doi:10.1258/ebm.2010.009258.

Page 25/27 
64. Whitfield J, Dy V, McQuilty R, et al. Genetic effects on toxic and essential elements in humans: arsenic, cadmium, copper, lead, mercury, selenium, and zinc in erythrocytes. Environ Health Perspect. 2010 Jun;118(6):776-82. doi:10.1289/ehp.0901541.

65. Rhodus NL. Zinc, impaired immunity, and oral disease in the geriatric patient. Gerodontics,1987 Aug;3(4):141-5.

66. Siddiqui M, Srivastava S, Mehrotra P. Environmental exposure to lead as a risk for prostate cancer. Biomed Environ Sci. 2002 Dec;15(4):298-305.

67. Pizent A, Colak B, Kljaković Z, et al. Prostate-specific antigen (PSA) in serum in relation to blood lead concentration and alcohol consumption in men. Arh Hig Rada Toksikol. 2009 Mar;60(1):69-78. doi:10.2478/10004-1254-60-2009-1901.

68. Zeng $X$, Jin $T$, Jiang $X$, et al. Effects on the prostate of environmental cadmium exposure-A crosssectional population study in China. Biometals. 2004;17(5):559-65.

69. Rapisarda V, Miozzi E, Loreto C, et al. Cadmium exposure and prostate cancer: insights, mechanisms and perspectives. Front Biosci (Landmark Ed). 2018 Mar;1:23:1687-700.

70. JuKun S, Dongbo Y, Jianguo Z, et al. Association Between Cd Exposure and Risk of Prostate Cancer: APRISMA-Compliant Systematic Review and Meta-Analysis. Medicine (Baltimore),2016,95(6): e2708

71. Waalkes MP. Cadmium carcinogenesis. Mutat Res. 2003;533:107-20. DOI:10.1016/j.mrfmmm.2003.07.011.

72. Edwin W, Eric A, Ganesh S. Prostate-specific antigen levels in relation to cadmium exposure and zinc intake: results from the 2001-2002 NHANES. The prostate, 2008(68):122-128.

73. Terrence $M$, Ock K, Chun. Zinc intake is associated with lower cadmium burden in US adults. The Journal of Nutrition. 2015 Dec;145(12):2741-8. doi:10.3945/jn.115.223099.

74. Zefferino R, Piccoli C, Ricciardi N, et al. Possible Mechanisms of Mercury Toxicity and Cancer Promotion: Involvement of Gap Junction Intercellular Communications and Inflammatory Cytokines. Oxid Med Cell Longev. 2017; 2017:7028583. doi: 10.1155/2017/7028583.

75. Mahmoud A, Al-Alem U, Dabbous F. Zinc Intake and Risk of Prostate Cancer: Case-Control Study and Meta-Analysis. PLoS One. 2016;11(11):e0165956. doi:10.1371/journal.pone.0165956.

\section{Figures}




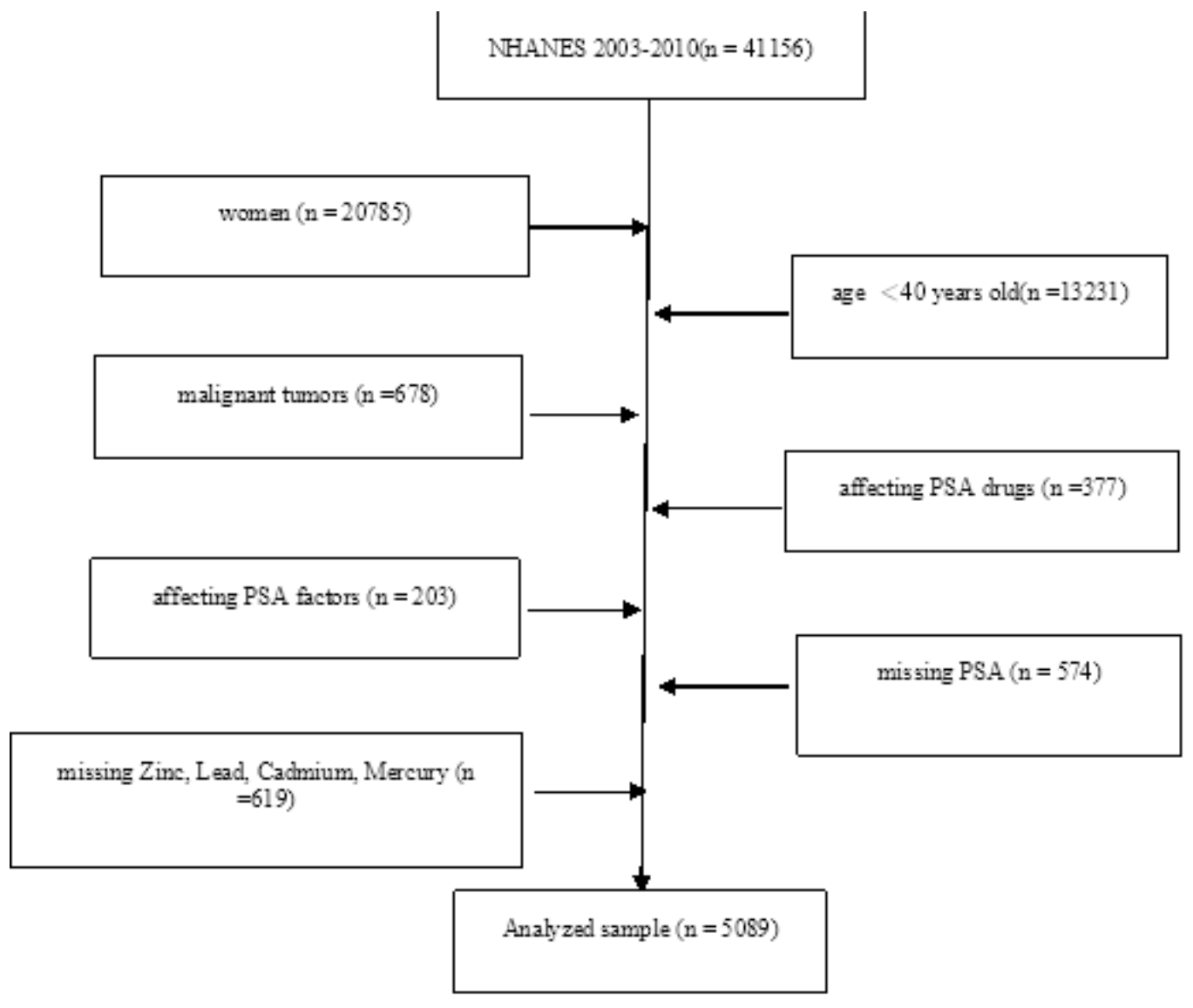

Figure 1

Flow chart of study participants. 